\title{
BIBECHANA
}

A Multidisciplinary Journal of Science, Technology and Mathematics

ISSN 2091-0762 (online)

Journal homepage: http://nepjol.info/index.php/BIBECHANA

\section{Approximation of a generalized Lipschitz class function by Euler - Cesàro means of Fourier series}

\author{
Binod Prasad Dhakal \\ Central Department of Education Mathematics \\ Tribhuvan University, Nepal \\ E-mail: binod_dhakal2004@yahoomail.com \\ Article history: Received 19 November, 2011; Accepted 27 November, 2012
}

\section{Abstract}

In this paper, I have taken product of two summability methods, Euler and Cesàro; and establish a new theorem on the degree of approximation of the function $\mathrm{f}$ belonging to $\mathrm{W}\left(\mathrm{L}^{\mathrm{p}}, \xi(\mathrm{t})\right)$ classes by Euler Cesàro method.

Key words and phrases: Degree of approximation; (E,1) (C,1) Summability; Fourier series.

\section{Definitions and Notations}

$$
\begin{aligned}
& \text { A function } \mathrm{f}(\mathrm{x}) \in \operatorname{Lip} \alpha \text {, if } \\
& |\mathrm{f}(\mathrm{x}+\mathrm{t})-\mathrm{f}(\mathrm{x})|=\mathrm{O}\left(|\mathrm{t}|^{\alpha}\right) \text { for } 0<\alpha \leq 1 \text { and } \mathrm{f} \in \operatorname{Lip}(\alpha, \mathrm{p}) \text {, if } \\
& \left(\int_{0}^{2 \pi}|\mathrm{f}(\mathrm{x}+\mathrm{t})-\mathrm{f}(\mathrm{x})|^{\mathrm{p}} \mathrm{dx}\right)^{\frac{1}{\mathrm{p}}}=\mathrm{O}\left(|\mathrm{t}|^{\alpha}\right), 0<\alpha \leq 1, \mathrm{p} \geq 1 .
\end{aligned}
$$

Given a positive increasing function $\xi(\mathrm{t}), \mathrm{p} \geq 1$, $\mathrm{f}(\mathrm{x}) \in \operatorname{Lip}(\xi(\mathrm{t}), \mathrm{p})$, if

$$
\begin{aligned}
& \left(\int_{0}^{2 \pi}|f(x+t)-f(x)|^{p} d x\right)^{\frac{1}{p}}=O(\xi(t)) \text { and } f \in W\left(L^{p}, \xi(t)\right), \text { if } \\
& \left(\int_{0}^{2 \pi}\left|(f(x+t)-f(x)) \sin ^{\beta} x\right|^{p} d x\right)^{\frac{1}{p}}=O(\xi(t)),(\beta \geq 0) .
\end{aligned}
$$

It is noted that, $\mathrm{W}\left(\mathrm{L}^{\mathrm{p}}, \xi(\mathrm{t})\right) \stackrel{\beta=0}{\longrightarrow} \operatorname{Lip}(\xi(\mathrm{t}), \mathrm{p}) \stackrel{\xi(\mathrm{t})=\mathrm{t}^{\alpha}}{\longrightarrow} \operatorname{Lip}(\alpha, \mathrm{p}) \stackrel{\mathrm{p} \rightarrow \infty}{\longrightarrow} \operatorname{Lip} \alpha$ So, $\operatorname{Lip} \alpha \subseteq \operatorname{Lip}(\alpha, p) \subseteq \operatorname{Lip}(\xi(t), p) \subseteq \mathrm{W}\left(\mathrm{L}^{\mathrm{p}}, \xi(\mathrm{t})\right)$ for $0<\alpha \leq 1$ and $\mathrm{p} \geq 1$. 
We define the norm \|\|$_{\mathrm{p}}$ by $\quad\|\mathrm{f}\|_{\mathrm{p}}=\left\{\int_{0}^{2 \pi}|\mathrm{f}(\mathrm{x})|^{\mathrm{p}} \mathrm{dx}\right\}^{\frac{1}{\mathrm{p}}}, \quad \mathrm{p} \geq 1$.

The degree of approximation $E_{n}(f)$ of function $f: R \rightarrow R$ is given by

$$
E_{n}(f)=\operatorname{Min}\left\|t_{n}-f\right\|_{p}
$$

Where $t_{n}$ is trigonometric polynomial of degree $n$ [2].

Let $\mathrm{f}$ be $2 \pi$ periodic, integrable over $(-\pi, \pi)$ in the sense of Lebesgue and belonging to $\mathrm{W}\left(\mathrm{L}^{\mathrm{p}}, \xi(\mathrm{t})\right)$ class, then its "Fourier series" is given by

$$
f(t)=\frac{1}{2} a_{o}+\sum_{n=1}^{\infty}\left(a_{n} \cos n t+b_{n} \sin n t\right) .
$$

Let $\sum_{n=0}^{\infty} u_{n}$ be the infinite series whose nth partial sum is given by $S_{n}=\sum_{i=0}^{n} u_{i}$.

The Cesåro means $(C, 1)$ of sequence $\left\{S_{n}\right\}$ is $\sigma_{n}=\frac{1}{n+1} \sum_{k=0}^{n} S_{k}$.

If $\sigma_{\mathrm{n}} \rightarrow \mathrm{S}$, as $\mathrm{n} \rightarrow \infty$ then sequence $\left\{\mathrm{S}_{\mathrm{n}}\right\}$ or the infinite series $\sum_{\mathrm{n}=0}^{\infty} \mathrm{u}_{\mathrm{n}}$ is said to be summable by Cesåro means method $(\mathrm{C}, 1)$ to $\mathrm{S}$. It is denoted by

$$
\sigma_{\mathrm{n}} \rightarrow \mathrm{S}(\mathrm{C}, 1) \text {, as } \mathrm{n} \rightarrow \infty[3] \text {. }
$$

The Euler means $(E, 1)$ of sequence $\left\{\mathrm{S}_{\mathrm{n}}\right\}$ is $\mathrm{E}_{\mathrm{n}}^{1}=\frac{1}{2^{\mathrm{n}}} \sum_{\mathrm{k}=0}^{\mathrm{n}}\left(\begin{array}{l}\mathrm{n} \\ \mathrm{k}\end{array}\right) \mathrm{S}_{\mathrm{k}}$

If $E_{n}^{1} \rightarrow S$ as, $n \rightarrow \infty$ then sequence $\left\{S_{n}\right\}$ or infinite series $\sum_{n=0}^{\infty} u_{n}$ is said to be summable by Euler means method $(E, 1)$ to $S$. It is denoted by

$$
E_{n}^{1} \rightarrow S(E, 1) \text {, as } n \rightarrow \infty[4] \text {. }
$$

The $\mathrm{E}_{\mathrm{n}}^{1}$ transformation of $\left\{\sigma_{\mathrm{n}}\right\}$ is denoted by $\mathrm{t}_{\mathrm{n}} \mathrm{E}_{1}, \mathrm{C}_{1}$, which is $(\mathrm{E}, 1)(\mathrm{C}, 1)$ transformation of $\left\{\mathrm{S}_{\mathrm{n}}\right\}$ and defined as $\mathrm{t}_{\mathrm{n}}^{\mathrm{E}_{1}, \mathrm{C}_{1}}=\frac{1}{2^{\mathrm{n}}} \sum_{\mathrm{k}=0}^{\mathrm{n}}\left(\begin{array}{l}\mathrm{n} \\ \mathrm{k}\end{array}\right) \sigma_{\mathrm{k}}=\frac{1}{2^{\mathrm{n}}} \sum_{\mathrm{k}=0}^{\mathrm{n}}\left(\begin{array}{l}\mathrm{n} \\ \mathrm{k}\end{array}\right) \frac{1}{\mathrm{k}+1} \sum_{\mathrm{r}=0}^{\mathrm{k}} \mathrm{S}_{\mathrm{r}}$.

If $\mathrm{t}_{\mathrm{n}}^{\mathrm{E}_{1}, \mathrm{C}_{1}} \rightarrow \mathrm{S}, \quad$ as $\mathrm{n} \rightarrow \infty$ then sequence $\left\{\mathrm{S}_{\mathrm{n}}\right\}$ or infinite series $\sum_{\mathrm{n}=0}^{\infty} \mathrm{u}_{\mathrm{n}}$ is said to be summable by (E,

1) $(C, 1)$ means method to $S$. It is denoted by

We use following notations.

$$
\mathrm{t}_{\mathrm{n}}^{\mathrm{E}_{1}, \mathrm{C}_{1}} \rightarrow \mathrm{S}(\mathrm{E}, 1)(\mathrm{C}, 1), \quad \text { as } \mathrm{n} \rightarrow \infty .
$$

$$
\begin{aligned}
& \phi(t)=f(x+t)+f(x-t)-2 f(x) \\
& N_{n}^{E_{1}, C_{1}}=\frac{1}{2^{n+1} \pi} \sum_{k=0}^{n}\left(\begin{array}{l}
n \\
k
\end{array}\right) \frac{\sin ^{2}(k+1) \frac{t}{2}}{(k+1) \sin ^{2} \frac{t}{2}}
\end{aligned}
$$




\section{Main Theorem}

In present paper, the degree of approximation of a function $f \in W\left(L^{p}, \xi(t)\right)$ class by $(E, 1)(C, 1)$ means of a Fourier series has been determined in the following form:

Theorem: If $\mathrm{f}: \mathrm{R} \rightarrow \mathrm{R}$ is $2 \pi$ periodic, Lebesgue integrable function in $(-\pi, \pi)$ and is $\mathrm{W}\left(\mathrm{L}^{\mathrm{p}}, \xi(\mathrm{t})\right)$, then the degree of approximation of function $\mathrm{f}$ by $(\mathrm{E}, 1)(\mathrm{C}, 1)$ means of Fourier series $(1)$ satisfies,

$$
\left\|\mathrm{t}_{\mathrm{n}}^{\mathrm{E}_{1}, \mathrm{C}_{1}}-\mathrm{f}\right\|_{\mathrm{p}}=\mathrm{O}\left((\mathrm{n}+1)^{\beta+\frac{1}{\mathrm{p}}} \xi\left(\frac{1}{\mathrm{n}+1}\right)\right) \text {, for } \mathrm{n}=1,2,3,4, \ldots \ldots \ldots \ldots
$$

Provided $\xi(\mathrm{t})$ satisfy the following conditions;

$$
\begin{gathered}
\left\{\frac{\xi(\mathrm{t})}{\mathrm{t}}\right\} \text { is monotonic decreasing } \\
\left\{\int_{0}^{\frac{1}{\mathrm{n}+1}}\left(\frac{\mathrm{t}|\phi(\mathrm{t})|}{\xi(\mathrm{t})}\right)^{\mathrm{p}} \sin ^{\beta \mathrm{p}} \mathrm{tdt}\right\}^{\frac{1}{\mathrm{p}}}=\mathrm{O}\left(\frac{1}{\mathrm{n}+1}\right), \\
\left\{\int_{\frac{1}{\mathrm{n}+1}}^{\pi}\left(\frac{\mathrm{t}^{-\delta}|\phi(\mathrm{t})|}{\xi(\mathrm{t})}\right)^{\mathrm{p}} \mathrm{dt}\right\}^{\frac{1}{\mathrm{p}}}=\mathrm{O}\left((\mathrm{n}+1)^{\delta}\right)
\end{gathered}
$$

where $\delta$ is an arbitrary number such that $\mathrm{q}(1-\delta)-1>0$, condition (5) and (6) hold uniformly in $\mathrm{x}$.

\section{Lemmas}

We need the following Lemmas for the proof of our theorem.

Lemma 1: Let

$$
\begin{aligned}
& \mathrm{N}_{\mathrm{n}}^{\mathrm{E}_{1}, \mathrm{C}_{1}}(\mathrm{t})=\frac{1}{2^{\mathrm{n}+1} \pi} \sum_{\mathrm{k}=0}^{\mathrm{n}}\left(\begin{array}{l}
\mathrm{n} \\
\mathrm{k}
\end{array}\right) \frac{\sin ^{2}(\mathrm{k}+1) \frac{\mathrm{t}}{2}}{(\mathrm{k}+1) \sin ^{2} \frac{\mathrm{t}}{2}} \text {, then } \\
& \mathrm{N}_{\mathrm{n}}^{\mathrm{E}_{1}, \mathrm{C}_{1}}(\mathrm{t})=\mathrm{O}(\mathrm{n}+1), \text { for } 0<\mathrm{t}<\frac{1}{\mathrm{n}+1} .
\end{aligned}
$$

Proof:

$$
\begin{aligned}
\mathrm{N}_{\mathrm{n}}^{\mathrm{E}_{1}, \mathrm{C}_{1}}(\mathrm{t}) & =\frac{1}{2^{\mathrm{n}+1} \pi} \sum_{\mathrm{k}=0}^{\mathrm{n}}\left(\begin{array}{l}
\mathrm{n} \\
\mathrm{k}
\end{array}\right) \frac{\sin ^{2}(\mathrm{k}+1) \frac{\mathrm{t}}{2}}{(\mathrm{k}+1) \sin ^{2} \frac{\mathrm{t}}{2}} \\
& \leq \frac{1}{2^{\mathrm{n}+1} \pi} \sum_{\mathrm{k}=0}^{\mathrm{n}}\left(\begin{array}{l}
\mathrm{n} \\
\mathrm{k}
\end{array}\right) \frac{(\mathrm{k}+1)^{2} \sin ^{2} \frac{\mathrm{t}}{2}}{(\mathrm{k}+1) \sin ^{2} \frac{\mathrm{t}}{2}} \\
& =\frac{1}{2^{\mathrm{n}+1} \pi} \sum_{\mathrm{k}=0}^{\mathrm{n}}\left(\begin{array}{l}
\mathrm{n} \\
\mathrm{k}
\end{array}\right)(\mathrm{k}+1)
\end{aligned}
$$


Binod Prasad Dhakal / BIBECHANA 9 (2013) 151-158 : BMHSS, p.154 (Online Publication: Nov., 2012)

$$
\begin{aligned}
& =\frac{1}{2^{n+1} \pi}\left[\sum_{k=0}^{n} k\left(\begin{array}{l}
n \\
k
\end{array}\right)+\sum_{k=0}^{n}\left(\begin{array}{l}
n \\
k
\end{array}\right)\right] \\
& =\frac{1}{2^{n+1} \pi}\left[n 2^{n-1}+2^{n}\right] \\
& =\left(\frac{n+2}{4 \pi}\right) \\
& \leq \frac{(n+1)}{2 \pi} \\
& =O(n+1)
\end{aligned}
$$

Lemma 2: Let $\mathrm{N}_{\mathrm{n}}^{\mathrm{E}_{1}, \mathrm{C}_{1}}$ be given as Lemma I, then

$$
\begin{aligned}
& \mathrm{N}_{\mathrm{n}}^{\mathrm{E}_{1}, \mathrm{C}_{1}}(\mathrm{t})=\mathrm{O}\left(\frac{1}{(\mathrm{n}+1) \mathrm{t}^{2}}\right), \text { for } \frac{1}{\mathrm{n}+1}<\mathrm{t}<\pi \\
&\left|\mathrm{N}_{\mathrm{n}}^{\mathrm{E}_{1}, \mathrm{C}_{1}}(\mathrm{t})\right| \leq \frac{1}{2^{\mathrm{n}+1} \pi} \sum_{\mathrm{k}=0}^{\mathrm{n}}\left(\begin{array}{l}
\mathrm{n} \\
\mathrm{k}
\end{array}\right) \frac{\left|\sin ^{2}(\mathrm{k}+1) \frac{\mathrm{t}}{2}\right|}{(\mathrm{k}+1)\left|\sin ^{2} \frac{\mathrm{t}}{2}\right|} \\
&=\frac{1}{2^{\mathrm{n}+1} \pi} \sum_{\mathrm{k}=0}^{\mathrm{n}}\left(\begin{array}{l}
\mathrm{n} \\
\mathrm{k}
\end{array}\right) \frac{|1-\cos (\mathrm{k}+1) \mathrm{t}|}{2(\mathrm{k}+1)\left|\sin ^{2} \frac{\mathrm{t}}{2}\right|} \\
& \leq \frac{1}{2^{\mathrm{n}+1} \pi} \sum_{\mathrm{k}=0}^{\mathrm{n}}\left(\begin{array}{l}
\mathrm{n} \\
\mathrm{k}
\end{array}\right) \frac{1}{(\mathrm{k}+1)\left|\sin ^{2} \frac{\mathrm{t}}{2}\right|} \\
&=\frac{\pi}{2^{\mathrm{n}+1} \mathrm{t}^{2}} \sum_{\mathrm{k}=0}^{\mathrm{n}}\left(\begin{array}{l}
\mathrm{n} \\
\mathrm{k}
\end{array}\right) \frac{1}{(\mathrm{k}+1)} \\
&=\frac{\pi}{2^{\mathrm{n}+1} \mathrm{t}^{2}}\left(\frac{2^{\mathrm{n}+1}-1}{\mathrm{n}+1}\right) \\
&=\frac{\pi}{(\mathrm{n}+1) \mathrm{t}^{2}}\left(1-\frac{1}{2^{\mathrm{n}+1}}\right) \\
& \leq \frac{\pi}{(\mathrm{n}+1) \mathrm{t}^{2}} \\
&=\mathrm{O}\left(\frac{1}{(\mathrm{n}+1) \mathrm{t}^{2}}\right)
\end{aligned}
$$

\section{Proof of the Theorem}

Following Titchmarsh [5], $\mathrm{n}^{\text {th }}$ partial sum of Fourier series (1) at $\mathrm{t}=\mathrm{x}$ is given by 
$\mathrm{S}_{\mathrm{n}}(\mathrm{x})-\mathrm{f}(\mathrm{x})=\frac{1}{2 \pi} \int_{0}^{\pi} \phi(\mathrm{t}) \frac{\sin \left(\mathrm{n}+\frac{\mathrm{t}}{2}\right) \mathrm{t}}{\sin \frac{\mathrm{t}}{2}} \mathrm{dt}$.

$(\mathrm{C}, 1)$ transform of $\mathrm{S}_{\mathrm{n}}$ i.e. $\sigma_{\mathrm{n}}$ is given by

$$
\begin{aligned}
& \frac{1}{\mathrm{n}+1} \sum_{\mathrm{k}=0}^{\mathrm{n}}\left(\mathrm{S}_{\mathrm{k}}(\mathrm{x})-\mathrm{f}(\mathrm{x})\right)=\frac{1}{2(\mathrm{n}+1) \pi} \int_{0}^{\pi} \frac{\phi(\mathrm{t})}{\sin \frac{\mathrm{t}}{2}} \sum_{\mathrm{k}=0}^{\mathrm{n}} \sin \left(\mathrm{k}+\frac{\mathrm{t}}{2}\right) \mathrm{t} d \mathrm{t} \\
& \sigma_{\mathrm{n}}(\mathrm{x})-\mathrm{f}(\mathrm{x})=\frac{1}{2(\mathrm{n}+1) \pi} \int_{0}^{\pi} \phi(\mathrm{t}) \frac{\sin ^{2}(\mathrm{n}+1) \frac{\mathrm{t}}{2}}{\sin ^{2} \frac{\mathrm{t}}{2}} \mathrm{dt} .
\end{aligned}
$$

Similarly, (E,1) transform of on i.e. $\mathrm{t}_{\mathrm{n}}^{\mathrm{E}_{1}, \mathrm{C}_{1}}$ is

$$
\begin{aligned}
& \frac{1}{2^{\mathrm{n}}} \sum_{\mathrm{k}=0}^{\mathrm{n}}\left(\begin{array}{l}
\mathrm{n} \\
\mathrm{k}
\end{array}\right)\left(\sigma_{\mathrm{k}}(\mathrm{x})-\mathrm{f}(\mathrm{x})\right)=\frac{1}{2^{\mathrm{n}+1} \pi} \int_{0}^{\pi} \phi(\mathrm{t}) \sum_{\mathrm{k}=0}^{\mathrm{n}}\left(\begin{array}{l}
\mathrm{n} \\
\mathrm{k}
\end{array}\right) \frac{\sin ^{2}(\mathrm{k}+1) \frac{\mathrm{t}}{2}}{(\mathrm{k}+1) \sin ^{2} \frac{\mathrm{t}}{2}} \mathrm{dt} \\
& \mathrm{t}_{\mathrm{n}}^{\mathrm{E}_{1}, \mathrm{C}_{1}}(\mathrm{x})-\mathrm{f}(\mathrm{x})=\int_{0}^{\pi} \phi(\mathrm{t}) \frac{1}{2^{\mathrm{n}+1} \pi} \sum_{\mathrm{k}=0}^{\mathrm{n}}\left(\begin{array}{l}
\mathrm{n} \\
\mathrm{k}
\end{array}\right) \frac{\sin ^{2}(\mathrm{k}+1) \frac{\mathrm{t}}{2}}{(\mathrm{k}+1) \sin ^{2} \frac{\mathrm{t}}{2}} \mathrm{dt} \\
&=\int_{0}^{\pi} \phi(\mathrm{t}) \mathrm{N}_{\mathrm{n}}^{\mathrm{E}_{1}, \mathrm{C}_{1}}(\mathrm{t}) \mathrm{dt} \\
&=\int_{0}^{\frac{1}{\mathrm{n}+1}} \phi(\mathrm{t}) \mathrm{N}_{\mathrm{n}}^{\mathrm{E}_{1}, \mathrm{C}_{1}}(\mathrm{t}) \mathrm{dt}+\int_{\frac{1}{\mathrm{n}+1}}^{\pi} \phi(\mathrm{t}) \mathrm{N}_{\mathrm{n}}^{\mathrm{E}_{1}, \mathrm{C}_{1}}(\mathrm{t}) \mathrm{dt} \\
&=\mathrm{I}_{1}+\mathrm{I}_{2}, \text { say. }
\end{aligned}
$$

For $\mathbf{I}_{1}$, applying Holder inequality and fact that $\phi(t) \in W\left(L^{p}, \xi(t)\right)$, we have

$$
\begin{aligned}
& \left|\mathrm{I}_{1}\right| \leq\left\{\int_{0}^{\frac{1}{\mathrm{n}+1}}\left(\frac{\mathrm{t}|\phi(\mathrm{t})|}{\xi(\mathrm{t})} \sin ^{\beta} \mathrm{t}\right)^{\mathrm{p}} \mathrm{dt}\right\}^{\frac{1}{\mathrm{p}}}\left\{\int_{0}^{\frac{1}{\mathrm{n}+1}}\left(\frac{\xi(\mathrm{t})}{\mathrm{t}^{\beta} \mathrm{sin}^{\beta}} \mathrm{N}_{\mathrm{n}}^{\mathrm{E}_{1}, \mathrm{C}_{1}}(\mathrm{t})\right)^{\mathrm{q}} \mathrm{dt}\right\}^{\frac{1}{\mathrm{q}}} \\
& =O\left(\frac{1}{n+1}\right)\left\{\int_{0}^{\frac{1}{n+1}}\left(\frac{\xi(\mathrm{t})(\mathrm{n}+1)}{\mathrm{t}^{\beta+1}}\right)^{\mathrm{q}} \mathrm{dt}\right\}^{\frac{1}{q}} \\
& =\mathrm{O}\left(\xi\left(\frac{1}{n+1}\right)\right)\left\{\int_{\varepsilon}^{\mathrm{n}^{\frac{1}{n+1}}} \mathrm{t}^{-\mathrm{q}(\beta+1)} \mathrm{dt}\right\}^{\frac{1}{q}}, \text { by the Mean Value Theorem, where } 0<\varepsilon<\frac{1}{\mathrm{n}+1} . \\
& =\mathrm{O}\left(\xi\left(\frac{1}{\mathrm{n}+1}\right)\right)\left\{\left(\frac{\mathrm{t}^{-\mathrm{q}(\beta+1)+1}}{-\mathrm{q}(\beta+1)+1}\right)_{\varepsilon}^{\frac{1}{\mathrm{n}+1}}\right\}^{\frac{1}{q}} \\
& =\mathrm{O}\left(\xi\left(\frac{1}{\mathrm{n}+1}\right)\right)\left\{(\mathrm{n}+1)^{(\beta+1) \mathrm{q}-1}\right\}^{\frac{1}{q}}
\end{aligned}
$$




$$
\begin{aligned}
& =O\left((n+1)^{\beta+1-\frac{1}{q}} \xi\left(\frac{1}{n+1}\right)\right) \\
& =O\left((n+1)^{\beta+\frac{1}{p}} \xi\left(\frac{1}{n+1}\right)\right) .
\end{aligned}
$$

For $\mathbf{I}_{\mathbf{2}}$, applying Holder's inequality and taking $\delta$ as an arbitrary number such that $\mathrm{q}(1-\delta)-1>0$, we have

$$
\begin{aligned}
& \left|I_{2}\right| \leq\left\{\int_{\frac{1}{n+1}}^{\pi}\left(\frac{t^{-\delta}|\phi(t)|}{\xi(t)} \sin ^{\beta} t\right)^{p} d t\right\}^{\frac{1}{p}}\left\{\int_{\frac{1}{n+1}}^{\pi}\left(\frac{\xi(t) N_{n}(t)}{t^{-\delta} \sin ^{\beta} t}\right)^{q} d t\right\}^{\frac{1}{q}} \\
& =\left\{\int_{\frac{1}{n+1}}^{\pi}\left(\frac{t^{-\delta}|\phi(t)|}{\xi(t)}\right)^{p} d t\right\}^{\frac{1}{p}}\left\{\int_{\frac{1}{n+1}}^{\pi}\left(\frac{\xi(t)}{t^{-\delta+\beta+2}(n+1)}\right)^{q} d t\right\}^{\frac{1}{q}} \\
& =\mathrm{O}\left((\mathrm{n}+1)^{\delta-1}\right)\left\{\int_{\mathrm{n}+1}^{\frac{1}{\pi}}\left(\frac{\xi\left(\frac{1}{\mathrm{y}}\right)}{\mathrm{y}^{\delta-\beta-2}}\right)^{\mathrm{q}}\left(-\frac{\mathrm{dy}}{\mathrm{y}^{2}}\right)\right\}^{\frac{1}{\mathrm{q}}} \\
& =O\left((n+1)^{\delta} \xi\left(\frac{1}{n+1}\right)\right) O\left[\left\{\int_{\frac{1}{\pi}}^{\mathrm{n}+1} \mathrm{y}^{\mathrm{q}(1+\beta-\delta)-2} \mathrm{dy}\right\}^{\frac{1}{q}}\right] \text { Using condition (4) } \\
& =O\left((n+1)^{\delta} \xi\left(\frac{1}{n+1}\right)\right) O\left[\left\{\left(\frac{y^{q(1+\beta-\delta)-1}}{q(1+\beta-\delta)-1}\right)_{\frac{1}{\pi}}^{n+1}\right\}\right] \\
& =\mathrm{O}\left((\mathrm{n}+1)^{\delta} \xi\left(\frac{1}{\mathrm{n}+1}\right)\right) \mathrm{O}\left[\left\{\frac{1}{\mathrm{q}(1+\beta-\delta)-1}\left((\mathrm{n}+1)^{\mathrm{q}(1+\beta-\delta)-1}-\left(\frac{1}{\pi}\right)^{\mathrm{q}(1+\beta-\delta)-1}\right)\right\}^{\frac{1}{q}}\right] \\
& =\mathrm{O}\left((\mathrm{n}+1)^{\delta} \xi\left(\frac{1}{\mathrm{n}+1}\right)\right) \mathrm{O}\left((\mathrm{n}+1)^{1+\beta-\delta-\frac{1}{q}}\right) \\
& =\mathrm{O}\left((\mathrm{n}+1)^{\beta+1-\frac{1}{q}} \xi\left(\frac{1}{\mathrm{n}+1}\right)\right) \\
& =\mathrm{O}\left((\mathrm{n}+1)^{\beta+\frac{1}{\mathrm{p}}} \xi\left(\frac{1}{\mathrm{n}+1}\right)\right) .
\end{aligned}
$$

By (9), (10) and (11), we have

$$
\begin{aligned}
\left|\mathrm{t}_{\mathrm{n}}^{\mathrm{E}_{1}, \mathrm{C}_{1}}-\mathrm{f}\right| & =\left((\mathrm{n}+1)^{\beta+\frac{1}{\mathrm{p}}} \xi\left(\frac{1}{\mathrm{n}+1}\right)\right) \\
\text { or } \quad\left\|\mathrm{t}_{\mathrm{n}} \mathrm{E}_{1}, \mathrm{C}_{1}-\mathrm{f}\right\|_{\mathrm{p}} & =\mathrm{O}\left\{\int_{0}^{2 \pi}\left((\mathrm{n}+1)^{\beta+\frac{1}{\mathrm{p}}} \xi\left(\frac{1}{\mathrm{n}+1}\right)\right)^{\mathrm{p}} \mathrm{dx}\right\}^{\frac{1}{\mathrm{p}}}
\end{aligned}
$$




$$
\begin{aligned}
& =O\left((n+1)^{\beta+\frac{1}{p}} \xi\left(\frac{1}{n+1}\right)\right)\left\{\int_{0}^{2 \pi} d x\right\}^{\frac{1}{p}} \\
& =O\left((n+1)^{\beta+\frac{1}{p}} \xi\left(\frac{1}{n+1}\right)\right) .
\end{aligned}
$$

This completes the proof of theorem.

\section{Corollaries}

Corollary 1: If $\beta=0$ and $\xi(t)=t^{\alpha}, 0<\alpha \leq 1$ then the degree of approximation of a $2 \pi$ periodic function $\mathrm{f}$ belonging to class $\operatorname{Lip}(\alpha, \mathrm{p})$ is given by

Proof:

$$
\begin{aligned}
\left\|\mathrm{t}_{\mathrm{n}}^{\mathrm{E}_{1}, \mathrm{C}_{1}}-\mathrm{f}\right\|_{\mathrm{p}} & =\mathrm{O}\left(\frac{1}{(\mathrm{n}+1)^{\alpha-\frac{1}{\mathrm{p}}}}\right) \\
\left\|\mathrm{t}_{\mathrm{n}}^{\mathrm{E}_{1}, \mathrm{C}_{1}}-\mathrm{f}\right\|_{\mathrm{p}} & =\mathrm{O}\left((\mathrm{n}+1)^{\beta+\frac{1}{\mathrm{p}}} \xi\left(\frac{1}{\mathrm{n}+1}\right)\right) \\
= & \mathrm{O}\left((\mathrm{n}+1)^{\frac{1}{\mathrm{p}}} \xi\left(\frac{1}{\mathrm{n}+1}\right)\right) \\
& =\mathrm{O}\left((\mathrm{n}+1)^{\frac{1}{\mathrm{p}}} \frac{1}{(\mathrm{n}+1)^{\alpha}}\right) \\
& =\mathrm{O}\left(\frac{1}{(\mathrm{n}+1)^{\alpha-\frac{1}{\mathrm{p}}}}\right) .
\end{aligned}
$$

Corollary 2: If $\mathrm{p} \rightarrow \infty$ in corollary 1 then the degree of approximation of a $2 \pi$ periodic function $\mathrm{f}$ belonging to class $\operatorname{Lip} \alpha \quad(0<\alpha<1)$ is given by

$$
\left\|\mathrm{t}_{\mathrm{n}}^{\mathrm{E}_{1}, \mathrm{C}_{1}}-\mathrm{f}\right\|_{\infty}=\mathrm{O}\left(\frac{1}{(\mathrm{n}+1)^{\alpha}}\right) \text {, for } 0<\alpha<1 .
$$

Remarks: An independent proof of corollary can be developed along the same line as the theorem. Example: Consider the infinite series,

$$
1-4 \sum_{\mathrm{n}=1}^{\infty}(-3)^{\mathrm{n}-1}
$$

The $(\mathrm{E}, 1)(\mathrm{C}, 1)$ means of the sequence $\left\{\mathrm{S}_{\mathrm{n}}\right\}$ is given by

$$
\begin{aligned}
\mathrm{t}_{\mathrm{n}}^{\mathrm{E}_{1}, \mathrm{C}_{1}} & =\frac{1}{2^{\mathrm{n}}} \sum_{\mathrm{k}=0}^{\mathrm{n}}\left(\begin{array}{l}
\mathrm{n} \\
\mathrm{k}
\end{array}\right) \sigma_{\mathrm{k}} \\
& =\frac{1}{2(\mathrm{n}+1)}\left(1-(-1)^{\mathrm{n}+1}\right) .
\end{aligned}
$$

The infinite series $(15)$ is neither $(C, 1)$ nor $(E, 1)$ summable. But from $(16)$, it is summable by $(E, 1)(C, 1)$ method. Therefore product summability $(\mathrm{E}, 1)(\mathrm{C}, 1)$ is more powerful than the individual methods $(\mathrm{C}, 1)$ 
Binod Prasad Dhakal / BIBECHANA 9 (2013) 151-158 : BMHSS, p.158 (Online Publication: Nov., 2012)

and $(\mathrm{E}, 1)$. Consequently, $(\mathrm{E}, 1)(\mathrm{C}, 1)$ means gives the better approximation than individual methods $(\mathrm{C}, 1)$ and $(\mathrm{E}, 1)$.

\section{References}

[1] B. E. Rhoades, Journal of Mathematics, 3(2003) 245-247.

[2] A .Zygmund: Trigonometric Series, Cambridge University Press (1959).

[3] G. H. Hardy: On the Summability of Fourier series, Proc. London Math. Soc.,12(1913) 365-372.

[4] G. H. Hardy : Divergent Series, The University Press, Oxford ( 1949).

[5] E. C. Titchmarsh.: The Theory of functions, Second Edition, Oxford (1939). 